\title{
Characteristics of the North Pacific Oscillation in CMIP5 Models in Relation to Atmospheric Mean States
}

\author{
MI-KYUNG SUNG \\ Yonsei University, Seoul, South Korea \\ CHANGHYUN YOO \\ Ewha Womans University, Seoul, South Korea \\ SANG-WOOK YEH \\ Hanyang University, ERICA, Ansan, South Korea \\ YU KOSAKA \\ The University of Tokyo, Tokyo, Japan \\ SOON-IL AN \\ Yonsei University, Seoul, South Korea
}

(Manuscript received 18 June 2019, in final form 20 January 2020)

\begin{abstract}
The North Pacific Oscillation (NPO), the second leading atmospheric mode in the North Pacific Ocean, is known to be responsible for climate variability and extremes in adjacent regions. The reproducibility of the NPO in climate models is thus a topic of interest for the more accurate prediction of climate extremes. By investigating the spatial characteristics of the NPO in models from phase 5 of the Coupled Model Intercomparison Project (CMIP5), this study reveals the intimate relationship between the NPO structure and the atmospheric mean states over the North Pacific. The majority of the models reasonably capture the meridional contrast of pressure anomalies, but the detailed horizontal characteristics of the NPO are found to differ among the models. Diagnostic analysis of 30 climate models and long-term observations suggest that systematic bias in the mean atmospheric baroclinicity over the North Pacific crucially affects the horizontal shape and zonal position of the NPO. In the models in which the climatological continental trough over the western North Pacific extends farther to the east, the NPO tends to be simulated farther to the east, strengthening its impact on the downstream climate. In contrast, when the climatological continental trough is reduced in size toward the west, the growth of the NPO is limited to the west, and its influence is weakened downstream. This relationship can be understood via the altered available potential and kinetic energy conversions that feed the total energy of the NPO, primarily stemming from the difference in the mean horizontal temperature gradient and stretching deformation of the mean horizontal wind.
\end{abstract}

\section{Introduction}

The North Pacific Oscillation (NPO), which manifests as a basin-scale meridional variation in the atmospheric mass over the North Pacific Ocean, has been known to dominate wintertime climate variability in adjacent regions, that is, East Asia and North America (Linkin and Nigam 2008; Rogers 1981). Pronounced interdecadal

Corresponding author: Dr. Changhyun Yoo, cyoo@ewha.ac.kr modulations of the NPO and its relationship with adjacent climates also have been reported (Pak et al. 2014; Sung et al. 2019; Wang et al. 2007; Yeh et al. 2018). For example, the relationship between the NPO and the East Asian climate was tenuous before the mid-1970s, until the correlation between the two became significant, exhibiting an abrupt change (Wang et al. 2007). In contrast, on the other side of the Pacific, the climatic influence of the NPO on downstream North America was substantial before the mid-1970s, but it weakened 
thereafter (Sung et al. 2019). The influence of the NPO has subsequently become dominant again in the downstream climate since the 2000s, further aggravating thermal and hydrological extremes in North America (Baxter and Nigam 2015; Lee et al. 2015; Lin et al. 2017). Strong thermal advection accompanied by the dipolar pressure anomalies of the NPO was revealed to strengthen cold snaps in central-eastern North America, while changes to storm tracks cause droughts or floods, particularly in the western coastal regions of North America (Linkin and Nigam 2008).

To understand the interdecadal variation of the NPO and its influence, recent studies have examined its energetics (Tanaka et al. 2016; Sung et al. 2019). In the conventional view, the gain in kinetic energy by transient eddies was speculated to substantially contribute to the maintenance of the NPO (Nakamura et al. 1987). However, a quantitative comparison of the energy processes related to the NPO has found that the largest proportion of the total energy is accounted for by conversion of available potential energy (APE) from the background state (Tanaka et al. 2016). The horizontal heat flux across the mean isotherms efficiently transfers APE from the mean flow into the anomalies, which in turn maintains the NPO against thermal damping caused by diabatic heating and transient eddies. In fact, the transient eddy momentum flux positively contributes to the maintenance of the NPO, while its heat flux acts as a damping by reducing atmospheric baroclinicity. Due to this offset, the net feedback by the transient eddies is of secondary importance (Tanaka et al. 2016). These characteristics of the NPO revealed by energetics analysis are associated with its vertical structure, which tilts southwestward with increasing height (Linkin and Nigam 2008; Tanaka et al. 2016). The tilted vertical structure of the NPO indicates that the geopotential height tendency induced by differential thermal advection across the steep background temperature gradient can act to maintain the NPO (Holton 2004). This means that spatiotemporal variation in the background flow can play an important role in determining characteristics of the NPO, such as its amplitude and horizontal structure. This was indeed the case for the recent resurgence of the NPO, which has brought intense climate extremes to North America (Sung et al. 2019).

The dominant influence of the NPO on dry and cold extremes in the adjacent regions of East Asia and North America requires further evaluation, particularly in relation to the reproducibility of the NPO relative to observations in major climate models within phase 5 of the Coupled Model Intercomparison Project (CMIP5). It has been found that the majority of climate models can portray either the amplitude or the geographical characteristics of the NPO but only one-half of the models are able to simulate both features successfully (Chen et al. 2018; Wang et al. 2019). It still remains to be answered why some climate models can simulate the representative characteristics of the NPO while others cannot. Recent advances in understanding of the NPO dynamics have encouraged us to address this question. In this study, we investigate the characteristics of the NPO with a focus on its zonal positioning because this aspect has been identified as a primary cause of the interdecadal variation in the climatic influence of the NPO around the Pacific rim (Sung et al. 2019).

In section 3, the horizontal characteristics of the NPO in the models are compared to observations while, in section 4 , we investigate the intrinsic features of the mean conditions of each model and their relationship with the NPO. Particular attention is given to the temperature gradient in the climatological background field over the North Pacific because it determines the efficiency of APE conversion. Upper-tropospheric mean wind characteristics are also investigated in relation to kinetic energy (KE) conversion, considering its positive contribution to the NPO (Tanaka et al. 2016). Evaluating the performance of current models and examining the exact role of model bias have important implications for future climate predictions. As predicted on the basis of various warming scenarios, global warming is expected to lead to a diverse range of changes in regional climates in terms of mean conditions and perturbation growth. Thus, understanding the characteristics of the NPO in relation to the climatological conditions within these models may hint at future changes in the behavior of the NPO, thus providing potentially useful information on climate extremes. A summary and discussion are presented in section 5 .

\section{Data and methods}

The characteristics of the NPO are investigated using 30 models from CMIP5 (Table 1; the numbering of models is for convenience and does not represent any order of superiority). To focus on the intrinsic characteristics of the NPO simulated in each model, we analyze the preindustrial control (piControl) experiment in the CMIP5, which incorporates radiative forcing fixed at preindustrial levels. As an observational reference, we use the National Oceanic and Atmospheric Administration/Cooperative Institute for Research in Environmental Sciences (NOAA/CIRES) Twentieth Century Reanalysis Project version-2c dataset (20CR) spanning the period 1850-2014, which was obtained online (https://www.esrl.noaa.gov/psd/) (Compo et al. 2011). Because the influence of the NPO is dominant during winter (Linkin and Nigam 2008), our analysis 
TABLE 1. List of 30 CMIP5 models and data lengths (in parentheses) of piControl experiments analyzed in this study. Expansions of model names can be found online (https://www.ametsoc.org./PubsAcronymList).

\begin{tabular}{clclcl}
\hline \hline Model no. & \multicolumn{1}{c}{ Model name } & Model no. & \multicolumn{1}{c}{ Model name } & Model no. & \multicolumn{1}{c}{ Model name } \\
\hline 1 & ACCESS1.0 (250 yr) & 11 & GFDL-ESM2M (500 yr) & 21 & IPSL-CM5B-LR (300 yr) \\
2 & BCC_CSM1.1(m) (400 yr) & 12 & GISS-E2-H-CC (251 yr) & 22 & MIROC5 (670 yr) \\
3 & BCC_CSM1.1 (500 yr) & 13 & GISS-E2-H (480 yr) & 23 & MIROC-ESM-CHEM (255 yr) \\
4 & CanESM2 (996 yr) & 14 & GISS-E2-R-CC (251 yr) & 24 & MIROC-ESM (531 yr) \\
5 & CESM1(BGC) (500 yr) & 15 & GISS-E2-R (225 yr) & 25 & MPI-ESM-LR (1000 yr) \\
6 & CESM1(WACCM) (200 yr) & 16 & HadGEM2-CC (240 yr) & 26 & MPI-ESM-MR (1000 yr) \\
7 & CMCC-CMS (500 yr) & 17 & HadGEM2-ES (575 yr) & 27 & MPI-ESM-P (1000 yr) \\
8 & CNRM-CM5 (850 yr) & 18 & INM-CM4.0 (500 yr) & 28 & MRI-CGCM3 (500 yr) \\
9 & FGOALS-s2 (501 yr) & 19 & IPSL-CM5A-LR (1000 yr) & 29 & NorESM1-ME (252 yr) \\
10 & GFDL-ESM2G (500 yr) & 20 & IPSL-CM5A-MR (300 yr) & 30 & NorESM1-M (501 yr) \\
\hline
\end{tabular}

focuses on seasonal mean variability of the NPO from December to the following February (DJF). Particular attention is given to two observational time periods, 1851-1900 (hereinafter P1) and 1951-2000 (P2), in comparison with the model results. Note that the number of observation stations available for $\mathrm{P} 1$ is smaller than for $\mathrm{P} 2$, which may undermine the reliability of the results for P1. However, the observational analysis is intended to capture the relationship between NPO characteristics and the background field on a planetary scale. It is also important to note that our selection of the period prioritizes statistical stability in NPO detection and changes in mean state. As such, the two 50-yr observational periods chosen for this study exhibit distinct behaviors in the focal relationship on an interdecadal time scale, but the results are robust for any reasonable changes to these periods.

The observational NPO pattern is defined as the second leading empirical orthogonal function (EOF2) of seasonal anomalies in sea level pressure (SLP) over the North Pacific region (Linkin and Nigam 2008). The same method is also applied to obtain the NPO in the CMIP5 models for $110^{\circ} \mathrm{E}-120^{\circ} \mathrm{W}, 20^{\circ}-70^{\circ} \mathrm{N}$. The corresponding principal component time series (PC2) was defined as the NPO index. This study focuses on the zonal position of the NPO's northern lobe and its relationship with the background state. The longitude of the northern center was determined on the basis of the mass-weighted center of the lowest $10 \%$ of SLP anomalies in the positive NPO pattern accompanied by anomalous low pressure to the north and (high pressure to the south, that is,

NPO northern center longitude $=\frac{\sum_{i} \operatorname{lon}(i) \operatorname{SLP}(i) \cos \theta}{\sum_{i} \mathrm{SLP}(i) \cos \theta}$,

where $i$ indicates a grid point within the lowest $10 \%$ of SLP anomalies over the North Pacific.
To understand the distinct characteristics of the NPO within the climate models, we compare the climatological mean states between the models that might affect the internal processes relevant to the maintenance of the NPO. To understand the dynamics of the NPO, the conversions of APE and KE from the background state to the anomalies and the difference between the models in the energy conversion arising from changes in the background conditions are analyzed following Tanaka et al. (2016) and Kosaka and Nakamura (2010):

$$
\begin{aligned}
& \text { APE conversion }=-\frac{R}{p S_{p}}\left(u^{\prime} T^{\prime} \frac{\partial \bar{T}}{\partial x}+v^{\prime} T^{\prime} \frac{\partial \bar{T}}{\partial y}\right) \text { and } \\
& \text { KE conversion }=\frac{v^{\prime 2}-u^{\prime 2}}{2}\left(\frac{\partial \bar{u}}{\partial x}-\frac{\partial \bar{v}}{\partial y}\right)-u^{\prime} v^{\prime}\left(\frac{\partial \bar{u}}{\partial y}+\frac{\partial \bar{v}}{\partial x}\right) .
\end{aligned}
$$

Then, vertical integration is employed. Unless noted otherwise, the APE and KE conversions indicate the values vertically integrated as follows:

$$
\frac{1}{g} \int_{900 \mathrm{hPa}}^{300 \mathrm{hPa}}(\mathrm{APE} \text { or KE conversion }) d p .
$$

Here, the overbar denotes the climatological seasonal mean during winter and the prime indicates seasonal anomalies associated with the NPO estimated by regression coefficients onto the NPO index. Also, $S_{p}$ $\left[\equiv R \bar{T} /\left(p C_{p}\right)-d \bar{T} / d p\right]$ is the static stability parameter, in which $R$ and $C_{p}$ denote the gas constant of dry air and specific heat at constant pressure, respectively. For the rest, we follow standard notations.

The statistical significance is determined using twosided Student's $t$ tests and bootstrap resampling method. The $t$ test is adopted to test significance of correlation or regression coefficient, while a bootstrap approach is to evaluate the robustness of the difference between CMIP5 model groups, by creating 1000 random subsets. 

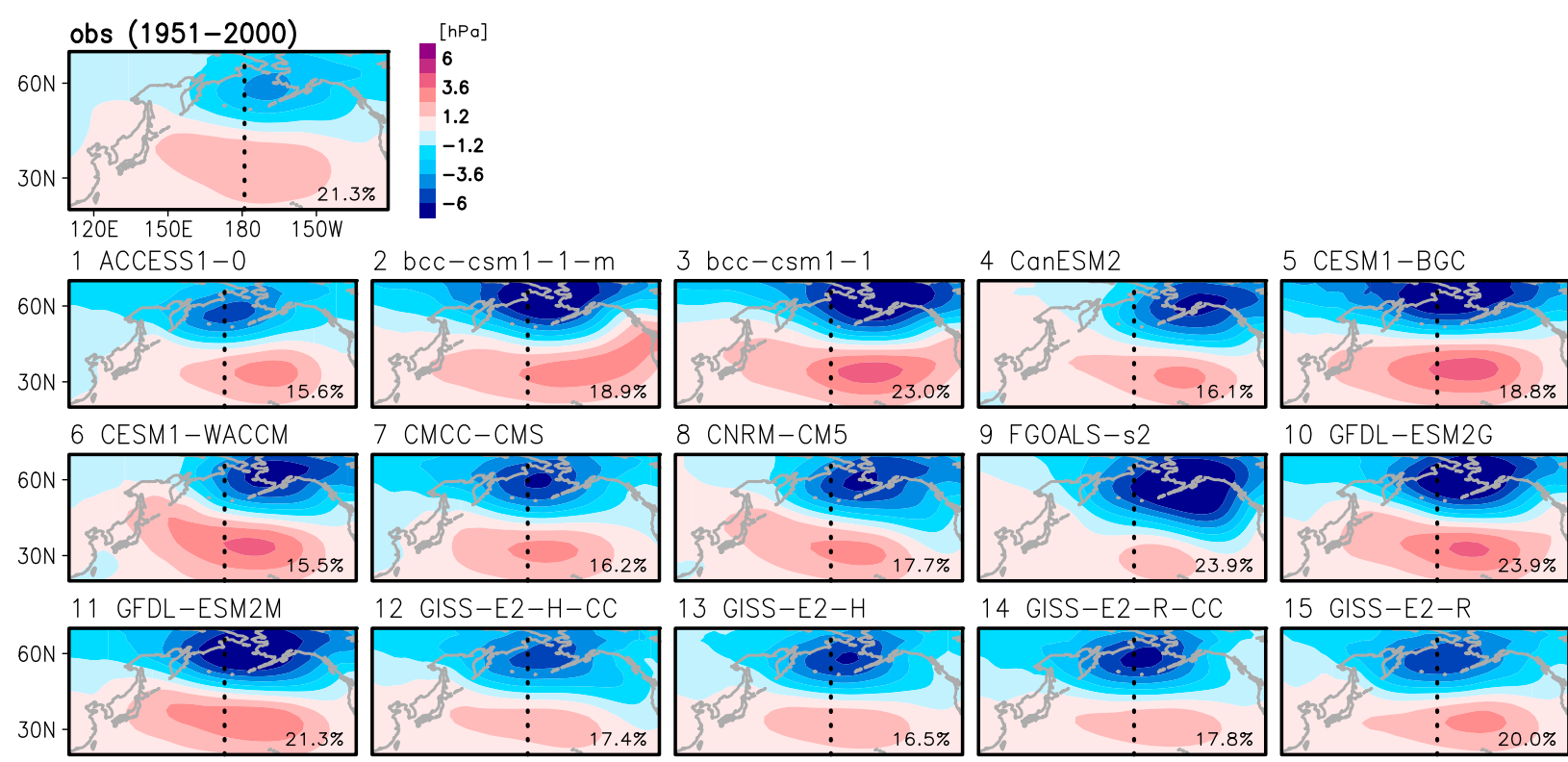

10 GFDL-ESM2G
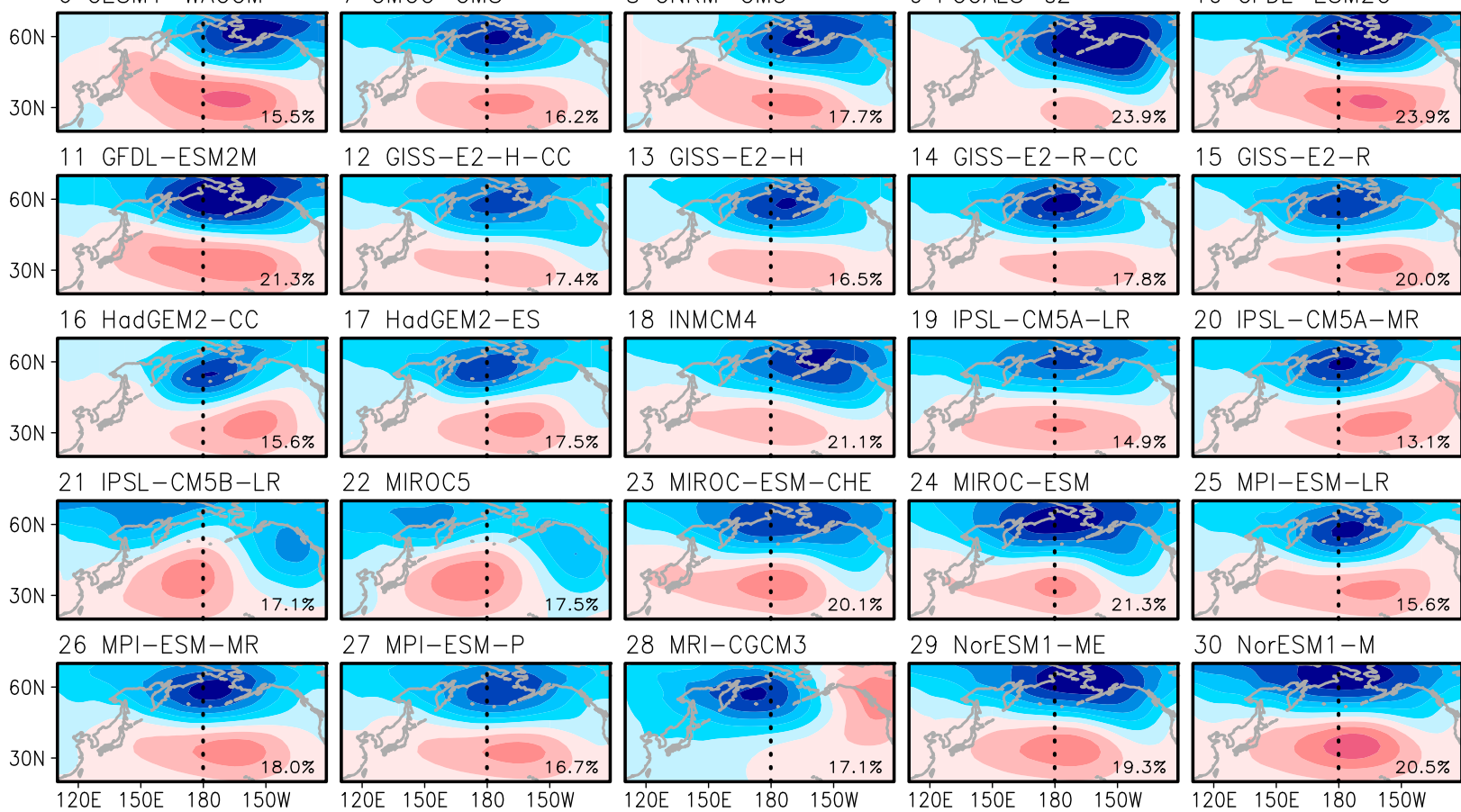

FIG. 1. EOF2 patterns of DJF seasonal mean SLP anomalies for the 1951-2000 observational period and piControl experiments of CMIP5 models. The numbers at the bottom right of the panels indicate the percentage of variance explained by the EOF2, and the black dotted line represents the date line.

To examine whether the NPOs of the two observational periods are significantly different from each other, we also performed an $F$ test by comparing the ratio of variances explained by the NPO.

\section{Spatial characteristics of the NPO in CMIP5 and observations}

As reported in previous studies, the NPO is generally simulated well by climate models (Chen et al. 2018; Wang et al. 2019). Figure 1 demonstrates that the meridional dipole structure of the NPO can be detected in most models as the second leading EOF of SLP anomalies, consistent with observations. Specifically, 27 of the EOF2 patterns from the 30 climate models successfully simulate the characteristic features of the NPO.
However, the proportion of the variance explained by the EOF2 patterns varies between the models. In IPSLCM5A-MR (model 20), for example, the corresponding EOF2 explains $13.1 \%$ of the total variance in pressure, which is far below that of the observations $(21.3 \%)$, while some models, such as FGOALS-s2 (model 9) and GFDL-ESM2G (model 10) reach figures of up to $23.9 \%$.

We find that three of the 30 models do not exhibit typical features of the NPO. The EOF2 of MRI-CGCM3 (model 28 ) displays an apparent zonal contrast in SLP anomalies, while IPSL-CM5B-LR (model 21) and MIROC5 (model 22) produce double northern centers of action with the same sign. It is notable that the EOF2 patterns can differ dramatically between models with a similar modeling setup, such as IPSL-CM5A-LR (model 19) and IPSLCM5B-LR (model 21), as well as MIROC5 (Model 22) 

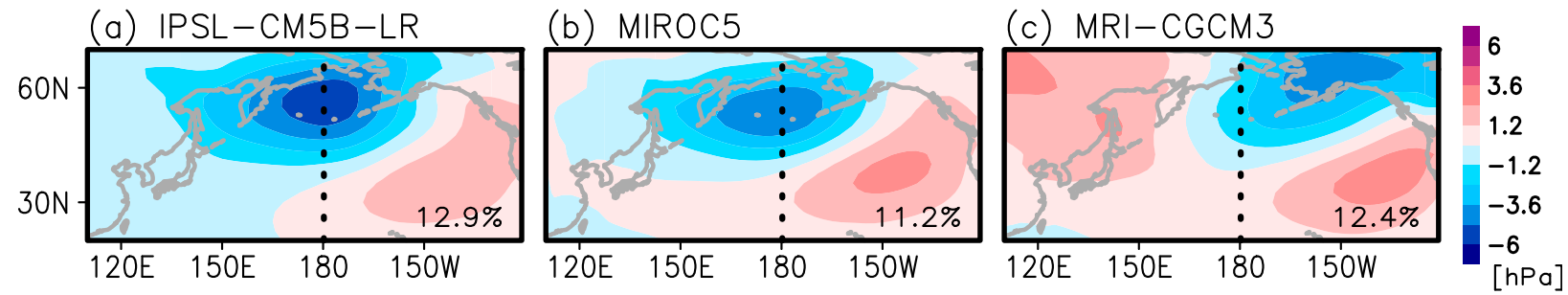

FIG. 2. The third EOF patterns of DJF seasonal mean SLP anomalies in (a) IPSL-CM5B-LR, (b) MIROC5, and (c) MRI-CGCM3. The numbers at the bottom right of the panels indicate the percentage of variance explained by EOF3, and the black dotted line represents the date line.

and MIROC-ESM (model 24). It is also interesting that historical simulations of IPSL-CM5B-LR and MIROC5 have revealed well-defined dipolar patterns in EOF2 (Wang et al. 2019), while that of MRI-CGCM3 has exhibited similar behavior to our findings (see also Wang et al.'s Fig. 1). In fact, in the piControl simulation of IPSLCM5B-LR and MIROC5, the meridional dipole structure of the NPO is captured as the third EOF (EOF3) pattern (Fig. 2). Although the northern and southern centers shift to the west and east, respectively, compared to the conventional NPO, the EOF3 patterns appear to reflect NPOlike variability in these two models. The manifestation of the NPO as an EOF3 pattern indicates that the NPO-like variability in these models is considerably weaker than in the other models, as further illustrated by the low variance fractions explained by EOF3 (12.9\% for IPSL-CM5BLR and $11.2 \%$ for MIROC5). In section 4, we will show that this variation in the results of the EOF analysis for these models and the weak variability in the NPO are associated with the characteristic features of the climatological background state. For MRI-CGCM3 (model 28 ), neither the EOF2 nor EOF3 patterns resemble the observed NPO (Fig. 2c). Therefore, we ignore MRICGCM3 when reexamining the simulated NPO patterns in comparison with the observations.

While the captured NPO patterns are roughly similar to the observations, there are differences in terms of the zonal position. For instance, CanESM2 (model 4) has its northern center over Alaska, while the center in ACCESS1.0 (model 1) appears in the middle of the North Pacific across the date line (the black dotted line in Fig. 1). For an objective comparison of the zonal position of the NPO between the models, the longitude of the NPO northern center is estimated using the EOF2 or EOF3 patterns (see the section 2). The bars in Fig. 3 represent the central longitude of the NPO in the models, ranging from $176.2^{\circ} \mathrm{E}$ to the west to $150.2^{\circ} \mathrm{W}$ to the east. In particular, the two models whose zonal position is estimated using the EOF3 (blue bars) exhibit the westernmost center position among the models.
The red bars in Fig. 3 represent the NPO patterns for P1 (1851-1900) and P2 (1951-2000), ${ }^{1}$ whose spatial patterns are given in Fig. 4. We find that the observed EOF2 patterns for the two periods differ dramatically in terms of their meridional dipole structure. The northern center of the NPO during P1 (Fig. 4a) is found over Alaska, which is farther to the east compared to P2 (Fig. 4b). The two EOF2 patterns also differ from that for the entire period (i.e., 1851-2013) (Fig. 4c). These changes in EOF2 patterns may partly be due to the changes in the number of observational stations incorporated for data periods of $\mathrm{P} 1$ and $\mathrm{P} 2$. However, the SLP anomalies regressed onto a single PC2 spanning the entire period exhibit consistent features (contours in Figs. $4 d$ and $4 \mathrm{e}$ ). Similar to the EOF2 obtained for P1 only, the SLP anomalies in the regression field exhibit a pronounced eastward shift in the northern center, a conspicuous difference from P2. These differences between the periods are unlikely to be a statistical artifact, because the variance of SLP anomalies explained by each regression pattern is significantly different between the periods (dotted area in Figs. $4 \mathrm{f}$ and $4 \mathrm{~g}$ ). Therefore, the SLP anomalies for each period reflect the actual spatial features of the NPO.

The change in the zonal position of the NPO between decades has important implications for adjacent regional climates because, when the NPO is located close to North America, it has greater influence on cold and warm extremes in the downstream region (Sung et al. 2019; Wang et al. 2007). Similarly, a shift in the NPO in the opposite direction results in a closer relationship with the East Asian winter climate. This can be clearly seen in the correlation map with surface air temperature (shading in Figs. 4d and 4e). The strong positive

\footnotetext{
${ }^{1}$ The $\mathrm{P} 1$ and $\mathrm{P} 2$ here are not defined exactly the same as in Sung et al. (2019), although the zonal variation in the NPO is robust in both cases. This is because, as also addressed in Sung et al. (2019), NPO variation occurs slowly on an interdecadal time scale.
} 


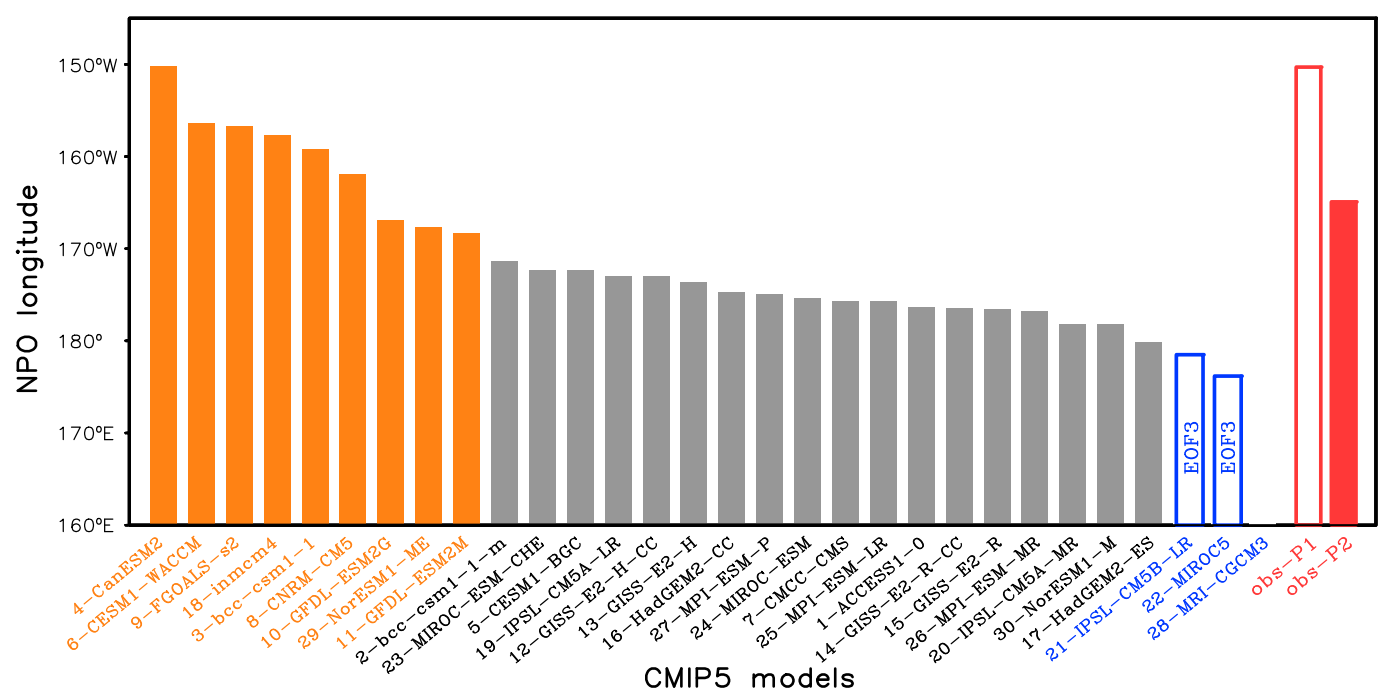

FIG. 3. Longitude of the NPO's northern center estimated from the EOF2 or EOF3 patterns of seasonal SLP anomalies in CMIP5 models except for MRI-CGCM3, which does not reproduce NPO-like variability. The orange and gray bars represent NPOeast and NPOwest models, respectively, based on the EOF2. The blue bars represent the values estimated from the EOF3 using IPSL-CM5B-LR and MIROC5 models. The red bars display observational data for P1 (1851-1900) and P2 (1951-2000).

correlation observed in North America during P1 weakens during P2, while the weak or neutral correlations over East Asia during P1 become significantly positive during P2.
To examine the relationship between the location of the NPO and its influence on the CMIP5 models, a similar analysis can be undertaken by classifying the models into "NPOeast" and "NPOwest" groups based
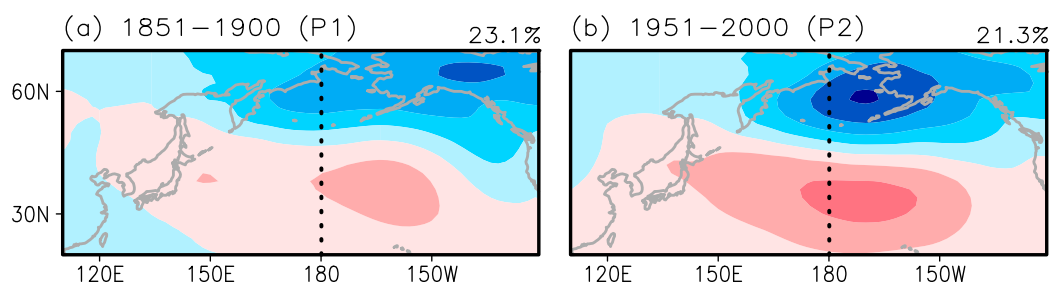

(c) $1851-2013$
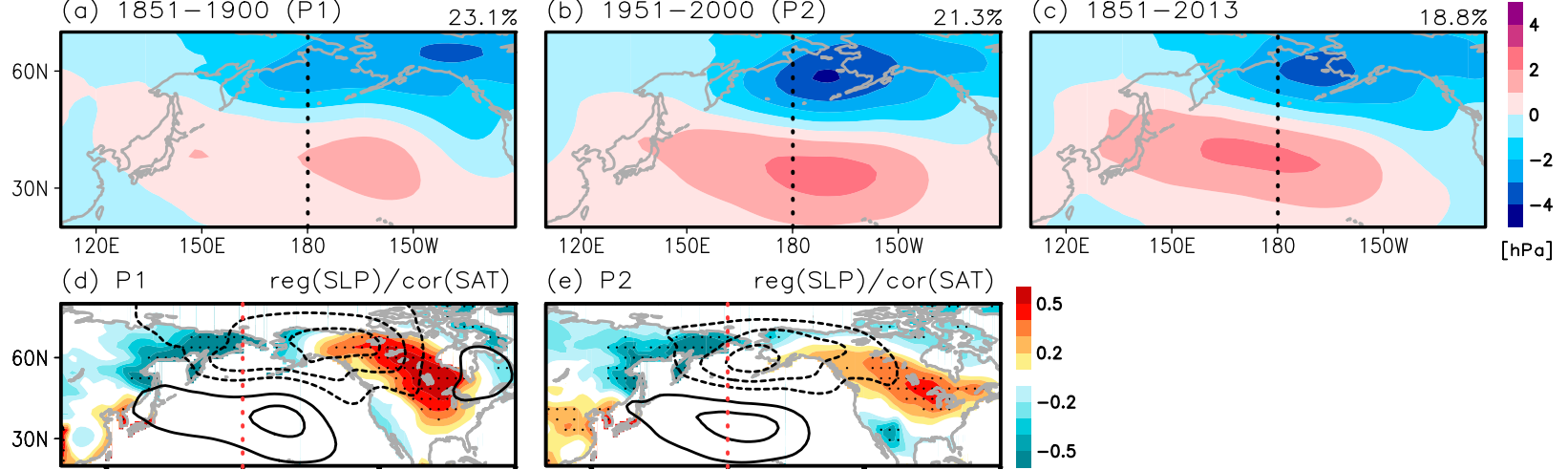

(f) $\operatorname{var}(P 1)$

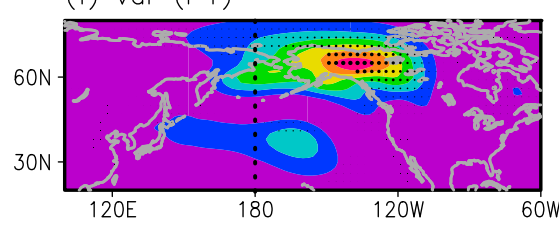

(g) $\operatorname{var}(P 2)$

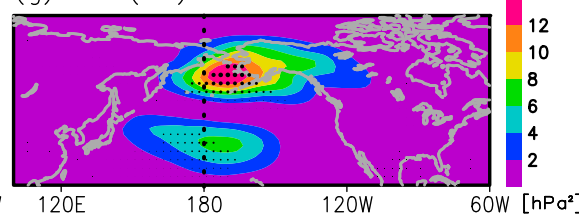

FIG. 4. Observational EOF2 patterns in seasonal SLP anomalies for (a) 1851-1900 (P1), (b) 1951-2000 (P2), and (c) 1851-2013 using 20CR data. The numbers in the upper right of the panels indicate the percentage of variance explained by the EOF2 in each period. Also shown are the SLP anomaly patterns (contours; 1-hPa intervals without zero lines) for (d) P1 and (e) P2, obtained by regression onto the PC2 of the entire period using $20 \mathrm{CR}$ data (i.e., PC2 for 1851-2013). Shading indicates the correlation with the land surface air temperature and the stippled region indicates a significant correlation at the 95\% confidence level based on the Student's $t$ statistic. In addition, the variance of the SLP anomalies explained by the NPO [using PC2 of 1851-2013, as for (d) and (e)] is shown, with the dots over shading denote the regions showing significantly larger variance at the $95 \%$ confidence based on the one-tailed $\mathrm{F}$ test [i.e., (f) $H_{a}: \sigma_{P 1}^{2}>\sigma_{P 2}^{2}$ and (g) $H_{a}: \sigma_{P 1}^{2}<\sigma_{P 2}^{2}$ ]. The black [in (a)-(c), (f), and (g)] and red [in (d) and (e)] dotted lines represent the date line. 
on the longitudinal location of the northern pole of the NPO. That is, we classify nine models whose northern lobes are centered east of $170^{\circ} \mathrm{W}$ into the NPOeast group (BCC_CSM1.1, CanESM2, CESM1(WACCM), CNRM-CM5, FGOALS-s2, GFDL-ESM2G, GFDLESM2M, INM-CM4.0, and NorESM1-ME, marked in orange in Fig. 3), and the other 18 models-except for IPSL-CM5B-LR and MIROC5-into the NPOwest group (marked in gray in Fig. 3). The NPOeast can be compared with the observed NPO of P1, and the NPOwest with that of P2. This classification is based on the distribution of bars in Fig. 3, which jumps between BCC_CSM1.1(m) (Model 2) and GFDL-ESM2M (Model 11), while there is no conspicuous difference between the gray-colored models. However, we note that the results are not significantly different even when different classification criteria are employed. The two models whose NPO is defined by EOF3 (IPSL-CM5BLR and MIROC5) are categorized as the westernmost NPO group and analyzed separately. This additional categorization is required because these two models exhibit distinct features in their climatological mean states, as discussed later.

Once the models are classified, the correlations with surface air temperature within the groups are investigated (Fig. 5). It is found that the NPOeast and NPOwest groups display correlation patterns that are very similar to those found for the observations, especially in North America. The strong positive correlations for the NPOeast models over North America (Fig. 5a) contrasts with the weak positive correlation for the NPOwest models (Fig. 5b). When the northern center of the NPO moves farther westward, as in IPSL-CM5B-LR and MIROC5, the area with positive correlations retreats over North America, but the influence on the west expands far into northeastern China and Japan (Fig. 5c). It is notable that the correlation becomes reversed in sign particularly over eastern North America for the westernmost NPO group. The altered correlations suggest that interannual variability of the winter temperature accounted for by the NPO largely varies between models according to zonal characteristics of the NPO simulated in models. The close match between the observations and model simulations again strongly indicates that the zonal position of the NPO is of great importance to its influence on adjacent regional climate.

\section{Intermodel differences in climatological mean states and their relationship with the NPO}

\section{a. Mean field in NPOeast and NPOwest models}

Maintenance of the NPO largely depends on the heat exchange between relatively warmer North Pacific and cooler Asian continent (Tanaka et al. 2016). The heat

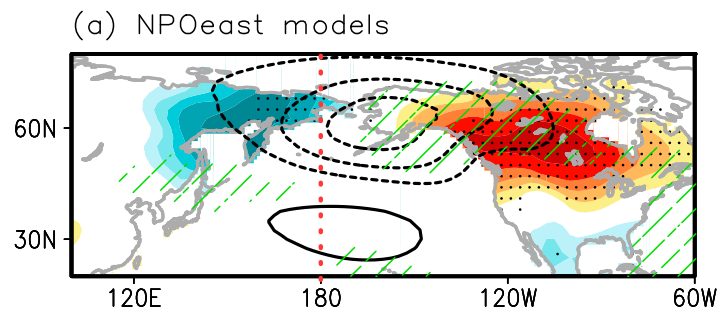

(b) NPOwest models

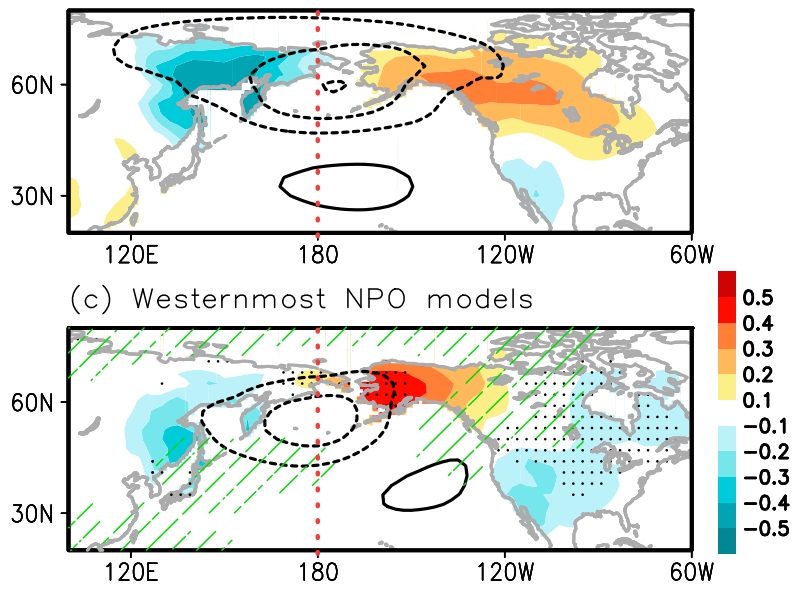

FIG. 5. As in Figs. 4d and 4e, but for the multimodel ensemble average, for (a) the NPOeast group, (b) the NPOwest group, and (c) the westernmost NPO models (IPSL-CM5B-LR and MIROC5). The model groups are categorized using the longitude of the NPO's northern center as given in Fig. 3 (contours; drawn at $\pm 2, \pm 4, \pm 6 \mathrm{hPa}$ ). The stippled and hatched areas indicate significant correlations of temperature and SLP anomalies, respectively, at the $95 \%$ confidence level. The red dotted line represents the date line.

flux across climatological isotherms can convert APE in the mean field into eddy APE $\left[\equiv R T^{2} /\left(2 p S_{p}\right)\right.$ at level $\left.p\right]$ because the phase line of the NPO tilts southwestward with height (Linkin and Nigam 2008; Tanaka et al. 2016). KE conversion from the Pacific jet is also revealed to contribute to the maintenance of the NPO, although such a process is less efficient than the APE conversion (Nakamura et al. 1987; Tanaka et al. 2016). These findings indicate that the growth of the NPO hinges on the intensity and horizontal structure of the background baroclinicity. Along the same line, Sung et al. (2019) demonstrated that spatial changes in the mean baroclinicity significantly affect the zonal position of the NPO by modulating the horizontal distribution of APE conversion. Their results showed that, when the mean temperature gradient intensifies to the east of the climatological maximum, additional APE conversion is facilitated via the NPO, which results in an eastward shift of the NPO. Building on this previous study, which examined temporal variation in background temperature in relation to the zonal position of 
(a) $\overline{\mathrm{T}} \&$ APE con $(700 \mathrm{hPa})$

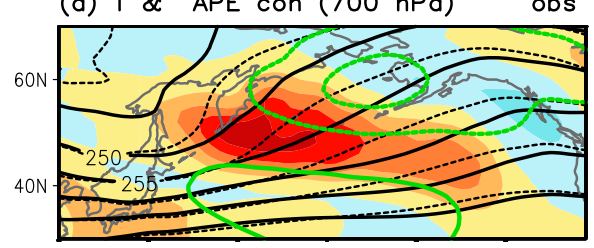

(c)

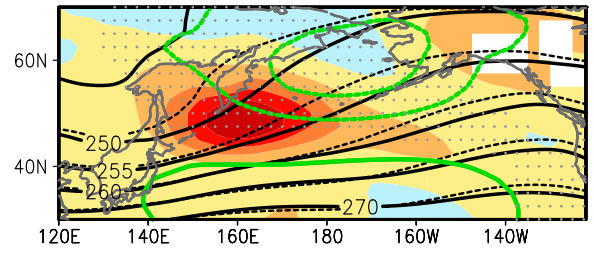

(b) $\bar{U} \& \mathrm{KE}$ con $(300 \mathrm{hPa})$

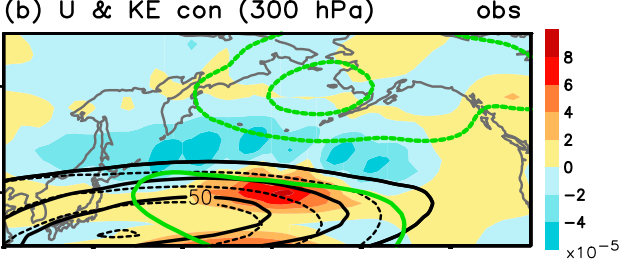

(d)
CMIP $\left[\mathrm{m}^{2} \mathrm{~s}^{-3}\right]$

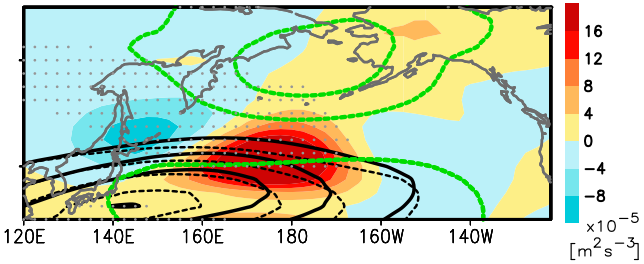

(e) $\left|\nabla T_{700}\right|\left(40-55^{\circ} \mathrm{N}\right.$ ave $)$

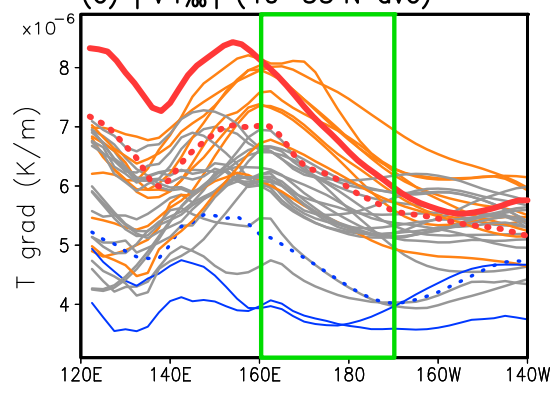

(f) $d U x-d V y\left(40-55^{\circ} \mathrm{N}\right.$ ave $)$

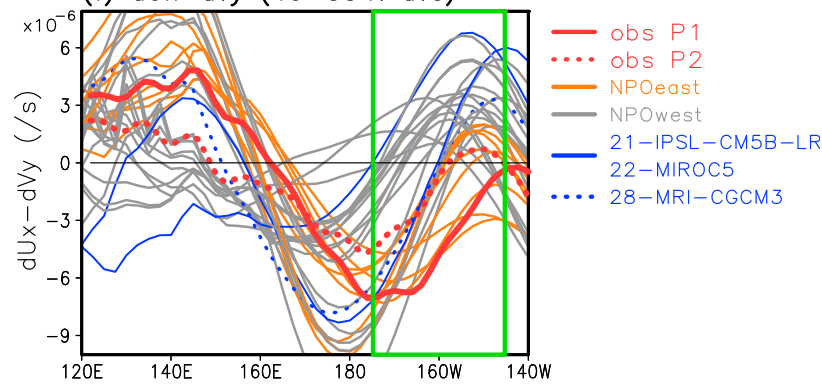

FIG. 6. (a) Isotherms for mean temperature (every $5 \mathrm{~K}$ ) for P1 (black solid lines) and P2 (black dashed lines), and APE conversion at $700 \mathrm{hPa}$ for $\mathrm{P} 2$ (shading). (b) Isotachs of mean zonal wind for P1 (black solid lines) and P2 (black dashed lines, drawn at 30,40, and $50 \mathrm{~m} \mathrm{~s}^{-1}$ ), along with $\mathrm{KE}$ conversion at $300 \mathrm{hPa}$ for P2 (shading). The green contours in (a) and (b) depict the SLP anomalies associated with the NPO patterns of P2 (1.5-hPa contour intervals without zero lines). (c),(d) Multimodel ensemble average of same quantities as in (a) and (b) but for the NPOeast group (black solid lines), the NPOwest group (the black dashed lines), and KE conversion of NPOwest group. The green contours in (c) and (d) depict SLP anomalies associated with the NPOwest group (drawn at $\pm 1, \pm 3, \pm 5$, and $\pm 7 \mathrm{hPa}$ ). The stippled area denotes significant difference of mean temperature [in (c)] and mean zonal wind [(d)] between the NPOeast and NPOwest groups. Also shown are individual curves of the zonal distributions of the (e) mean temperature gradient and (f) mean wind deformation in the $40^{\circ}-55^{\circ} \mathrm{N}$ range for each model. Orange, gray, and blue represent the NPOeast, NPOwest, and westernmost NPO models, respectively. The blue dotted line represents the mean temperature gradient for MRI-CGCM3 (model 28). Red solid and dotted lines denote values for observational $\mathrm{P} 1$ and $\mathrm{P} 2$ periods, respectively.

the NPO, we adopt the same method in this study to understand the specific characteristics of the NPOs in the CMIP5 models.

Before an intermodel comparison of the relationship between the background field and the zonal position of the NPO, we first examine the observational mean states of the $\mathrm{P} 1$ and $\mathrm{P} 2$ periods as an observational reference. Black solid contours in Fig. 6a, depicting a southwestnortheast slant of isotherms over the North Pacific, illustrate the shape of the continental trough along the eastern coast of Eurasia and the oceanic ridge during P1, while dashed contours represent that for P2. As indicated by green contours, the NPO grows over the region with a steep thermal gradient in the background field between the climatological trough and the ridge. This relative position of the NPO facilitates the transfer of
APE in the background flow to the anomaly field. Shading in Fig. 6a presents the actual horizontal distribution of APE conversion related to the NPO during P2 at lower troposphere $(700 \mathrm{hPa})$ where APE conversion is efficient. It can be seen that the APE conversion prevails on the southwestern flank of the NPO's northern lobe across the slanted isotherms. This result is consistent with findings by Tanaka et al. (2016).

We also analyze the difference in the mean temperature field between P1 and P2 (Fig. 6a). Relative to P2 (dashed contours), the isotherms of P1 (solid contours) expand farther eastward on the eastern flank of the continental trough. One can see that the decadal difference in the background flow between P1 and P2 dominates over the region of maximum APE conversion. This suggests that the zonal expansion/shrinkage of 
the climatological trough determines the thermal gradient, which can in turn affect the efficiency of APE conversion. Consistent to the observations, the NPOeast and NPOwest models also show similar difference in the horizontal structure of the continental trough (Fig. 6c). In other words, the mean isotherms of the NPOeast models (solid lines) illustrate a pronounced eastward expansion of the climatological trough, compared to that of the NPOwest group (dashed lines). The difference between the two groups is found to be significant (dots).

For a quantitative comparison of the background conditions, we measured the climatological temperature gradient $(|\nabla \bar{T}|)$ (Fig. 6e). The curves in Fig. 6e represent the zonal distribution of the mean temperature gradient averaged over $40^{\circ}-55^{\circ} \mathrm{N}$, where APE conversion is dominant. The models in the NPOeast and NPOwest groups are indicated by orange and gray curves, respectively. The curves overall show steep eastward increase of the temperature gradient between $140^{\circ}$ and $160^{\circ} \mathrm{E}$, and then gradually decrease to the east. This zonal structure of the mean temperature gradient matches with the distribution of APE conversion, which maximizes near the peak gradient region $\left(160^{\circ} \mathrm{E}\right)$ as shown in Figs. 6a and 6c. The collocation between the maximized APE conversion, mean temperature gradient, and the NPO demonstrates that the eddy heat flux can efficiently extract APE from the mean state across strong temperature gradient. Thus, when located away from the peak, NPO anomalies lose their efficiency of APE conversion. In this context, it is noteworthy that the temperature gradient curves of the NPOeast and NPOwest groups are overall relatively distinct from each other, and the differences between the groups are the largest over the zonal range of $160^{\circ} \mathrm{E}-170^{\circ} \mathrm{W}$ (marked by green rectangular in Fig. 6e). Consistently, in the observation, P1 (the period during which the NPO shifted to the east; solid red curve) is associated with enhanced mean temperature gradient than that of P2 (dotted red curve) within the same region. The eastward enhanced temperature gradient in the mean state of the NPOeast group presumably provides favorable condition for the NPO to shift farther eastward, which is addressed further in detail in section $4 \mathrm{~b}$.

Modulations by background flow through barotropic processes are also investigated. Figures $6 \mathrm{~b}$ and $6 \mathrm{~d}$ illustrate mean zonal wind at $300 \mathrm{hPa}$ (black contours), strongest over the southern edge of the trough. It can be seen that $\mathrm{KE}$ conversion at $300 \mathrm{hPa}$ (shading) is dominant over the northern diffluent region of the jet. This spatial pattern points out that favorable conditions for eddies to draw KE from the mean wind are located in the region of strong shear. We note that the total amount of $\mathrm{KE}$ conversion integrated for the entire troposphere is much weaker than that of APE conversion, although the $\mathrm{KE}$ conversion in the upper troposphere seems comparable to the APE conversion in the lower troposphere. Nonetheless, the altered mean wind between NPOeast and NPOwest models has important implications for the manifestation of the NPO, which will be further described in section 4c. Relative to observational P2 and the NPOwest group (dashed contours in Figs. 6b and $6 \mathrm{~d}$, respectively), mean zonal wind of $\mathrm{P} 1$ and the NPOeast group (solid contours in Figs. 6b and 6d, respectively) appears to expand to the north around jet exit region. This characteristic feature of mean wind is apparently accompanied with zonal expansion of the continental trough in the NPOeast group.

More detailed characteristics of the mean wind are illustrated in Fig. 6f. Here, we particularly compare the stretching deformation of wind, appearing in the first term of the KE conversion (i.e., $\partial \bar{u} / \partial x-\partial \bar{v} / \partial y$ ), which dominates the $\mathrm{KE}$ conversion in the northern jet exit region (Tanaka et al. 2016). The curves present the zonal distribution of the mean wind stretching deformation averaged over $40^{\circ}-55^{\circ} \mathrm{N}$, as in Fig. 6e. In most of the models and in the observations, mean wind deformation stay negative near the date line. Here, we need to note that negative values of mean wind deformation contribute positively to the growth of the NPO, as the KE conversion term is determined by multiplication with $v^{\prime 2}-u^{\prime 2}$, which is mostly negative in the concerned region. This explains why KE conversion is positive within the confined region only, while the APE conversion appears positive over wider regions of the North Pacific. In the east of the date line (green rectangle in Fig. 6f), the mean wind deformation begins to increase and becomes positive in some models. Notably, the mean wind deformation of NPOwest group models (gray curves) tends to increase more rapidly than that of NPOeast models (orange curves). The distinct slopes of mean wind deformation reveal that the background flow conditions of NPOeast models can supply KE to the NPO farther in the east than that of NPOwest. Detailed influence of the changes in the mean temperature and wind fields on the NPO is now assessed in terms of APE and KE conversions.

\section{b. APE conversion}

Influence of the background change on APE conversion can be estimated as follows. Because APE conversion is the multiplication of the eddy heat flux and mean temperature gradient (section 2), the conversion depends on the circulation pattern of the NPO. To quantify the specific influence of background change on APE conversion, the heat flux of the NPO should be fixed to a constant value. That is, the mean temperature gradients of each period are multiplied by the same $u^{\prime} T^{\prime}$ 
APE con under $\overline{\mathbf{T}}$ (P1)

(a) by heat flux of NPOeast

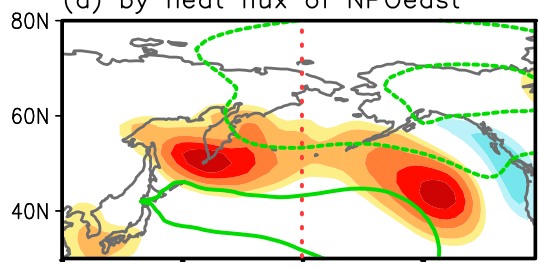

(d) by heat flux of NPOwest

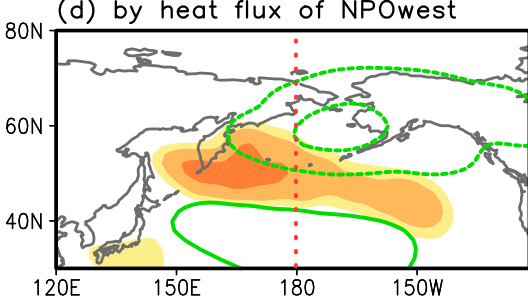

APE con under $\bar{T}$ (P2)

(b) by heat flux of NPOeast

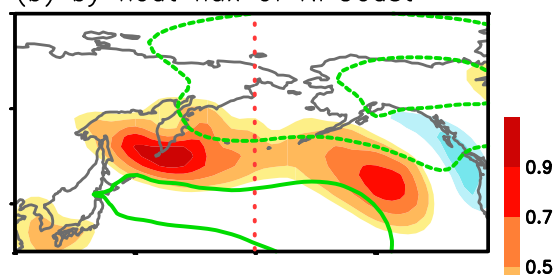

(e) by heat flux of NPOwest

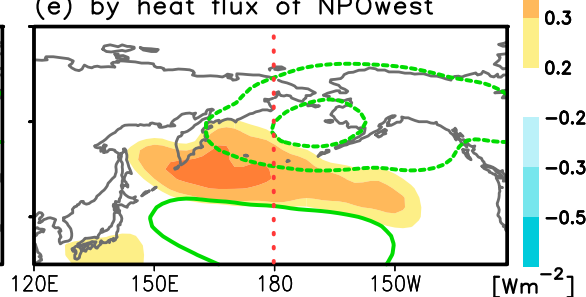

(c) diff by $\Delta \bar{T}$

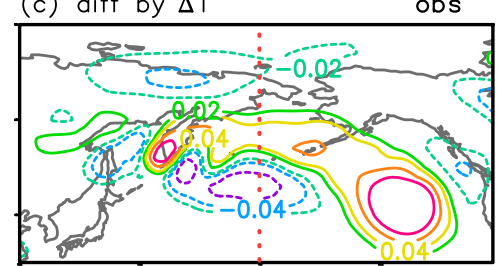

(f) diff by $\Delta \bar{T}$

FIG. 7. Vertically integrated APE conversions (shading) for (a),(d) P1 and (b),(e) P2 estimated using identical heat flux anomalies of the observed (top) NPOeast pattern (i.e., the NPO anomalies of P1) and (bottom) NPOwest pattern (i.e., the NPO anomalies of P2), as denoted by the green contours (SLP anomalies drawn at \pm 1.5 and $\pm 3 \mathrm{hPa}$ ). (c),(f) Difference between (a) and (b) [(a) minus (b)] or (d) and (e), respectively, displaying the influence of changes in the mean temperature gradient between P1 and P2. The contour interval is $0.02 \mathrm{~W} \mathrm{~m}^{-2}$.

and $v^{\prime} T^{\prime}$, and then the vertical integral is taken. By calculating the difference between chosen observational periods, the background modulation on APE conversion can be estimated. In the strict sense, however, this estimation is not a precise way to assess the influence of background change. This is because the altered background state would promote growth or decay of the NPO, which can lead to further changes in the energy conversion as well as heat and momentum budgets. As this evolutionary process of the NPO is not reflected in the estimates of fixed circulation anomalies, the background modulation might be underestimated in our results. Yet, we can expect that the conversion difference qualitatively captures how the background changes act to change the NPO pattern.

We find that the estimated APE conversion shows notable changes depending on the background mean condition (Fig. 7). Figures 7a and 7b present two APE conversion patterns (shading) that are obtained by using the same heat fluxes $u^{\prime} T^{\prime}$ and $v^{\prime} T^{\prime}$ of the observed NPOeast pattern (i.e., NPO during P1; green contours), but with different background mean states. It is apparent that the conversion patterns remain almost unchanged throughout the periods, as the APE conversion efficiency is largely dependent on the anomalous heat flux pattern. However, the differences between the two (Fig. 7c) illustrate the changes in APE conversion solely driven by altered background conditions. The positive values illustrate that the background flow during P1 serves to enhance the APE conversion further to the northeast compared to that of P2. Similar northeast enhancement can be also seen when the heat fluxes of the NPOwest pattern are employed (Figs. 7d-f). For both cases, the magnitude of the difference in APE conversion is as much as around $10 \%$ of its total value. It is notable that the pattern of APE conversion changes is consistent with the actual manifestations of the NPO. Namely, the APE conversion enhanced to the northeast due to background flow characteristics of P1 coincides with northeastward shift of the NPO during P1. Therefore, this coincidence suggests that the shift of the NPO between P1 and P2 is attributable to some extent to decadal variation in the mean temperature field over the North Pacific.

Now we compare the influence of background conditions in CMIP5 models. To this end, we first calculate average heat flux pattern of the NPOeast group (corresponding to green curves in upper panels of Fig. 8) and multiply it to inherent climatological mean temperature gradient in each model to estimated APE conversion (namely, APE conversion in 30 CMIP5 models is estimated based on the same NPO patterns). Then, composite average is respectively performed for the NPOeast and NPOwest groups (Figs. 8a,b). Consequently, their difference reveals the influence of distinct background conditions in the NPOeast and NPOwest groups (Fig. 8c), as was for the observational results (Fig. 7). Results are overall consistent to the observations; total APE conversion is dominant over the southwestern flank of the NPO (Fig. 8a) reflecting the features in the lower 
APE con under $\bar{T}_{\text {east }}$

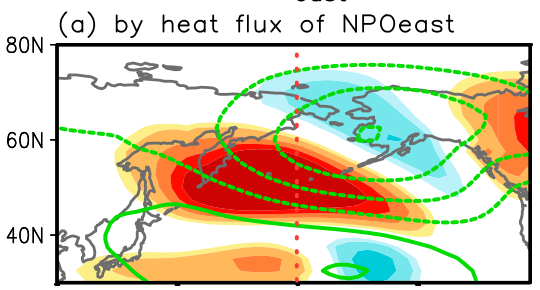

(d) by heat flux of NPOwest

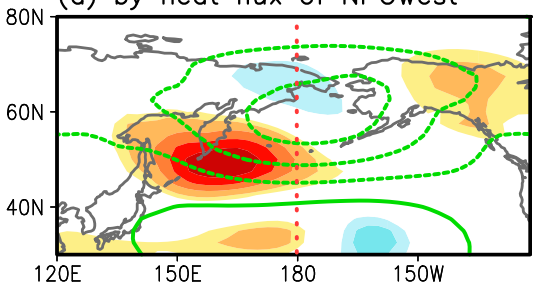

APE con under $\bar{T}_{\text {west }}$

(b) by heat flux of NPOeast

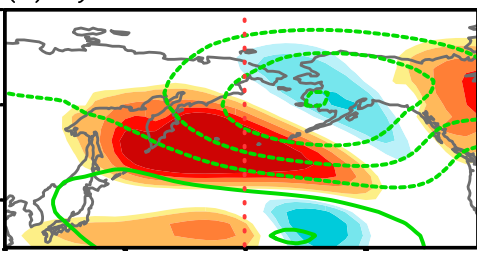

(e) by heat flux of NPOwest

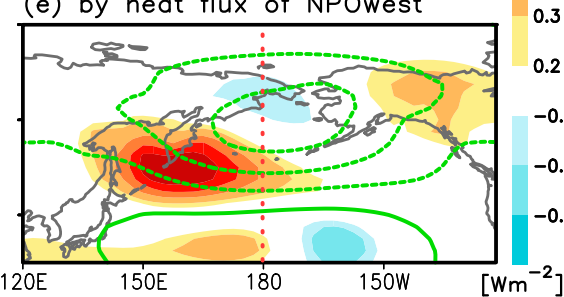

(c) diff by $\Delta \bar{T}$

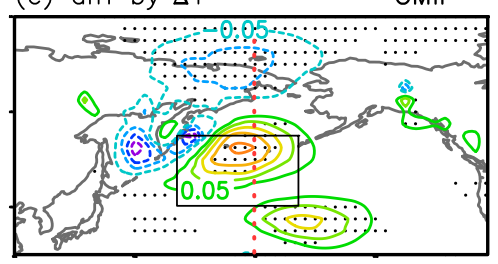

(f) diff by $\Delta \overline{\mathrm{T}} \quad$ CMIP

FIG. 8. Vertically integrated APE conversions (shading) corresponding to the NPO estimated for CMIP5 models. All quantities are the same as in Fig. 7 but denote multimodel ensemble average of NPOeast and NPOwest groups. See the text for detailed calculation (section $4 \mathrm{~b}$ ). The stippled area in (c) and (f) denotes significant difference of APE conversion between the NPOeast and NPOwest groups. The SLP anomalies are drawn for values at $\pm 1, \pm 3, \pm 5$, and \pm 7 in (a), (b), (d), and (e), and the contour interval for APE conversion difference in (c) and (f) is $0.05 \mathrm{~W} \mathrm{~m}^{-2}$.

troposphere as shown in Fig. 6c. The stronger APE conversion in the model compared to the observation is related to the stronger NPO anomalies (see Fig. 1; Chen et al. 2018). The difference map (Fig. 8c) shows zonal contrast of positive (to the east) and negative values (to the west). This eastward enhancement of APE conversion is significant at the $95 \%$ confidence level (dots) and serves to facilitate eastward shift of the NPO. It is noteworthy that the increase of APE conversion is pronounced particularly over the boxed region, which coincides with the region denoted by the green rectangle in Fig. 6e. The stronger background baroclinicity in the NPOeast models in this region is inferred to contribute to the enhancement of APE conversion.

On the other hand, when adopting the heat flux anomaly of the NPOwest pattern (green curves in bottom panels of Fig. 8), we find that the APE conversion difference between the NPOeast and NPOwest groups becomes tenuous (Figs. 8d-f), particularly in the same boxed region in Fig. 8c. This result indicates that the background flow of the NPOeast group may not generate eastward enhancement in the APE conversion in a certain situation, depending on changes in the heat fluxes. This can be inferred for an example of growing NPO anomalies that the background flow of the NPOeast group may not act to modulate position of the NPO during a certain stage of the growth, even while the APE conversion still serves to intensify the NPO. However, if some changes appear in the position of the NPO, APE conversion can accelerate the change as can be supposed from the result of Fig. 8c. We elucidate how the changes can be initiated in the following section.

\section{c. KE conversion}

Here we take a close look at the influence of background flow condition in terms of KE conversion. To this end, similar calculations to the APE conversion are performed using momentum fluxes (i.e., $v^{\prime 2}, u^{\prime 2}$, and $u^{\prime} v^{\prime}$ ) and mean wind stretching deformation instead of heat flux and mean temperature gradient (see section 2). First, using observation, KE conversion according to the mean wind deformation of P1 and P2 and their difference are depicted in Figs. 9a-c, by utilizing momentum flux corresponding to the observational NPOeast pattern, as a counterpart of results in Figs. 7a-c. Weaker efficiency of KE conversion than the APE conversion is already reported by Tanaka et al. (2016) (note an order-smaller magnitude in the color bar in Fig. 9 relative to that of APE conversion). However, we find that the difference induced by altered background wind deformation is considerable (Fig. 9c) to its APE counter part (Fig. 7c). Also, as for the APE conversion, the horizontal distribution of KE conversion difference shows zonal contrast of negative (to the west) and positive (to the east) values. This implies that the background wind deformation of P1 facilitates eastward shift of the NPO by altering KE conversion. The results are almost the same even if the momentum flux is replaced into that of the observational NPOwest pattern (Figs. 9d-f). 
KE con under $\overline{\vec{v}}(P 1)$

(a) by mtm flux of NPOeast

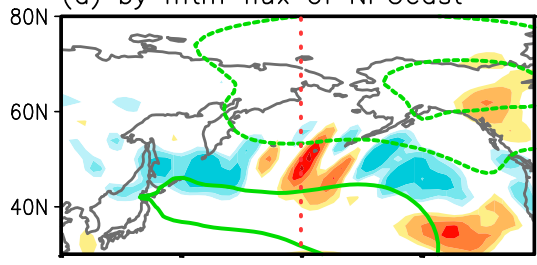

(d) by mtm flux of NPOwest

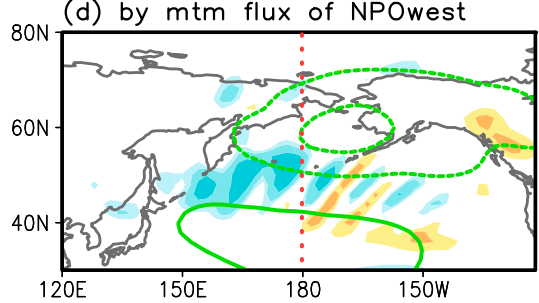

KE con under $\overline{\vec{v}}$ (P2)

(b) by $\mathrm{mtm}$ flux of NPOeast

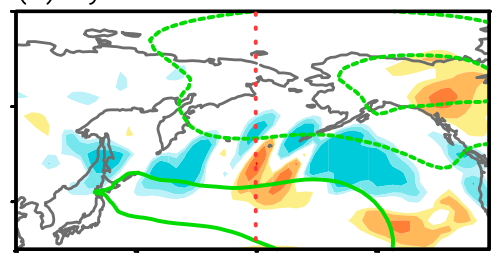

(e) by mtm flux of NPOwest

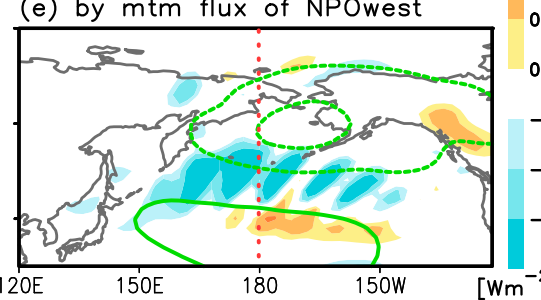

(c) diff by $\Delta \overline{\vec{V}}$

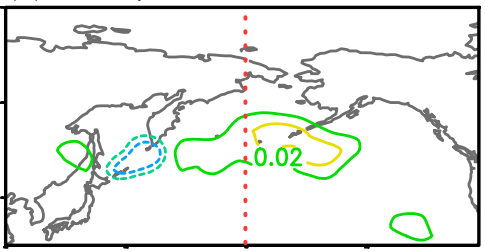

(f) diff by $\Delta \overrightarrow{\vec{V}}$

obs

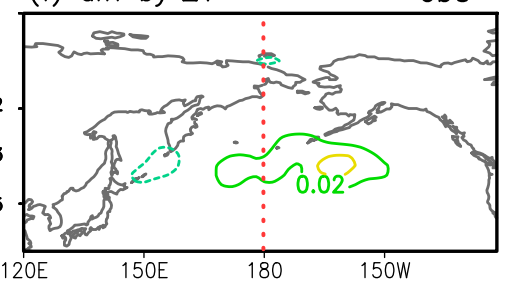

FIG. 9. As in Fig. 7, but for KE conversions (shading) estimated using mean wind stretching deformation and fixed momentum flux anomalies.

We can find consistent results also in CMIP5 models (Fig. 10). The positive KE conversion that is confined near the date line (Figs. 10a,b,d,e) shows pronounced zonal contrast between the model groups (Figs. 10c,f). The intensity and horizontal structure of KE conversion are unchanged irrespective of momentum flux pattern (i.e., comparison between the top and bottom rows). This was not the case for the APE conversion (Fig. 8). Interestingly, enhancement of $\mathrm{KE}$ conversion is pronounced particularly over the boxed region where the mean wind deformations are distinct between the NPOeast and NPOwest groups as shown in Fig. 6f (the box in Fig. 10c denotes the same region indicated by the green rectangle in Fig. 6f). Therefore, the significant enhancements of KE conversion to the east is inferred to result from the difference in the background wind deformation, and support significant modulation of the mean states on the zonal shift of the NPO for CMIP5 models.

It seems that the background flow affects the NPO both through alterating the KE and APE conversions. This relation between background flow and NPO characteristics can be verified in Fig. 11. For quantitative comparison of mean flow, first, the climatological temperature gradient $(|\nabla \bar{T}|)$ are averaged over the eastern marginal region of continental trough, that is, the
KE con under $\overline{\vec{v}}_{\text {east }}$

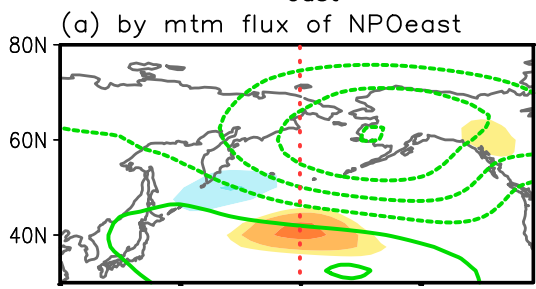

(d) by mtm flux of NPOwest

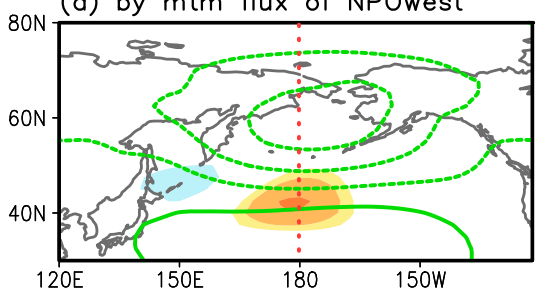

KE con under $\overline{\vec{V}}_{\text {west }}$

(b) by $\mathrm{mtm}$ flux of NPOeast

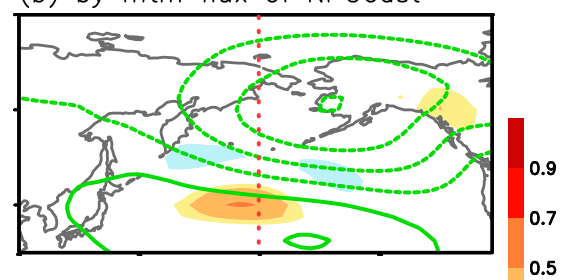

(e) by $\mathrm{mtm}$ flux of NPOwest

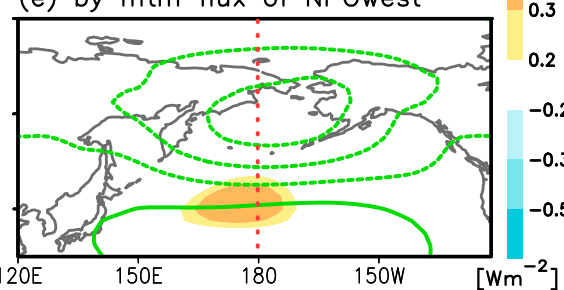

(c) diff by $\Delta \overline{\vec{V}}$

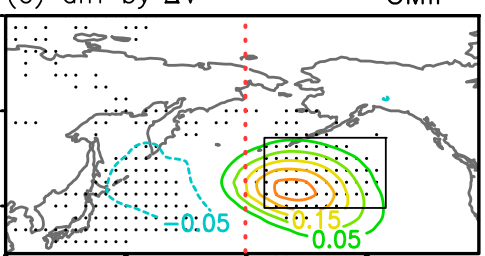

(f) $\operatorname{diff}$ by $\Delta \overline{\vec{V}}$

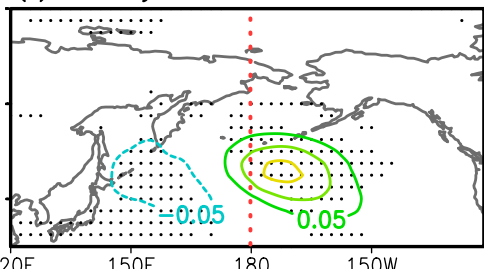

FIG. 10. As in Fig. 8, but for KE conversions in CMIP5 models (shading) estimated using mean wind stretching deformation and fixed momentum flux anomalies. 

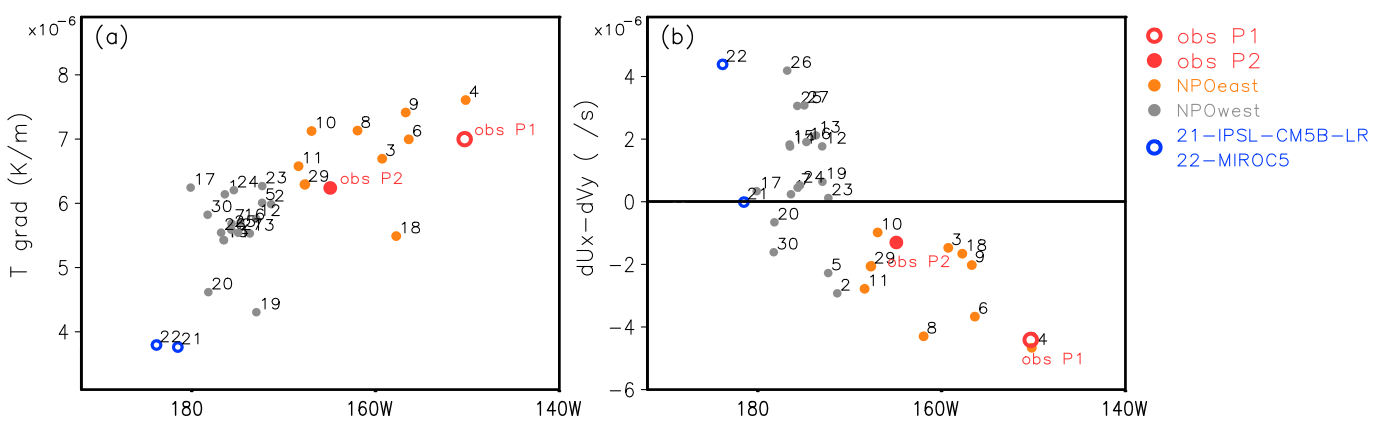

FIG. 11. (a) Relationship between the NPO's center longitude and climatological mean temperature gradient (averaged over $160^{\circ}-170^{\circ} \mathrm{E}, 40^{\circ}-55^{\circ} \mathrm{N}$; the black rectangular region in Fig. $8 \mathrm{c}$ ) in the CMIP5 models and the observational $\mathrm{P} 1$ and $\mathrm{P} 2$ periods. (b) As in (a), but for climatological mean wind stretching deformation $\partial \bar{u} / \partial x-\partial \bar{v} / \partial y$ averaged over $175^{\circ}-145^{\circ} \mathrm{W}, 40^{\circ}-55^{\circ} \mathrm{N}$ (the black rectangular region in Fig. 10c).

rectangular region in Fig. 6e (also in Fig. 8c). Figure 11a presents the zonal positions of the NPO in the CMIP5 models as a function of the mean temperature gradient. The central longitudes of the models are the same as those in Fig. 3. An overall linear relationship with mean temperature gradient is found (the intermodel correlation coefficient is 0.74 , which is significant at the $99 \%$ confidence level), which indicates the significant modulation of the zonal position of the NPO by background state baroclinicity, particularly by that over the eastern margin of climatological trough. It is worth noting that IPSL-CM5B-LR (model 21) and MIROC5 (model 22), which have the westernmost northern centers of the NPO estimated using EOF3, have the weakest mean atmospheric baroclinicity (the blue open circles). In contrast, CanESM2 (model 4) has the easternmost center of the NPO among the models, whose longitude is as far eastward as that of the observed NPO pattern during P1 (red open circle). Notably, the background baroclinicity of this model is the strongest of the models. In addition, the mean flow baroclinicity in P1 is consistently stronger than that in P2 (red closed circle). Overall, the NPOeast models (orange circles) have higher mean temperature gradients than the NPOwest models (gray circles) over the central North Pacific. Only one model, INM-CM4.0 (model 18), is an exception, with a mean atmospheric baroclinicity that is almost comparable to that of the NPOwest group, while the center longitude of the NPO is located farther to the east. The consistency between the models and observations supports the notion that the background flow conditions have a modulation effect on the NPO through the APE conversion.

The background modulation through KE conversion is also compared between models (Fig. 11b), by assessing mean wind stretching deformation in the first term in KE conversion, $\partial \bar{u} / \partial x-\partial \bar{v} / \partial y$, over the region of $175^{\circ}-145^{\circ} \mathrm{W}, 40^{\circ}-55^{\circ} \mathrm{N}$ (rectangular in Figs. 6f and $10 \mathrm{c}$ ).
The area is chosen considering significant background modulation through KE conversion therein. Figure 11b presents the zonal positions of the NPO as a function of the mean wind deformation at $300 \mathrm{hPa}$. As in the results of mean temperature gradient in Fig. 11a, intermodel comparison of mean wind deformation shows overall linear relation with zonal position of the NPO (the intermodel correlation is -0.75). All of the NPOeast group models (orange circles) have negative mean wind deformation, while a large number of NPOwest models (gray circles) have positive deformation. This distinction of NPOeast and NPOwest group models supports the background modulation to the NPO characteristic through the KE conversion. Similar to the results of mean temperature gradient, MIROC5 (model 22) is found to have unfavorable background flow condition in terms of mean wind deformation for eastward shift of the NPO (the highest blue open circle). In contrast, CanESM2 (model 4) has the strongest negative mean wind deformation, which can act to drive the NPO to grow farther to the east. The observed mean wind condition during P1 (red open circle) also shows values that are comparable to those of the easternmost model.

By comparing the results of APE and KE conversions of the models, we can draw a picture of how background flow modulates the zonal position of the NPO. As shown in section $4 \mathrm{~b}$, background flow of NPOeast group can provide eastward enhancement of APE conversion, but it may not for the NPO anomalies growing over the western North Pacific. Through KE conversion, meanwhile, the background flow of NPOeast group constantly drives NPO anomalies to develop in the east (e.g., Fig. 10f). One may speculate, thus, that the resultant changes of the NPO anomalies due to KE conversion would in turn lead to further shifting of the NPO via efficient processes of APE conversion. It is inferred that the modulations of background flow on both APE and KE conversions complementarily affect the positioning 
IPSL-CM5B-LR (model 21)

(a) $T 700 \& \nabla T$ diff

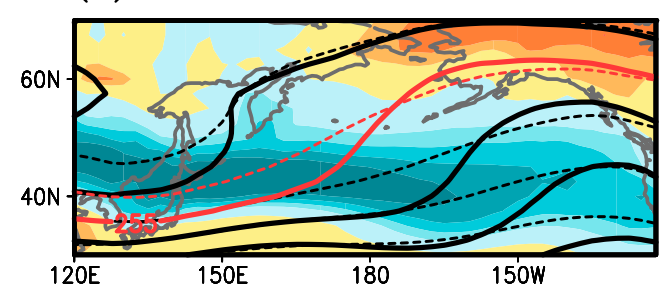

(c) $U 300 \& d U x-d V y$

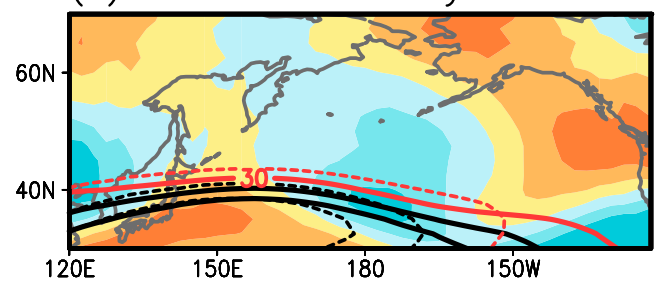

MIROC5 (model 22)

(b)

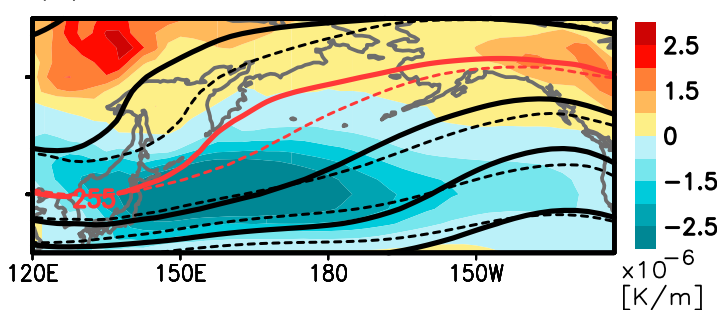

(d)

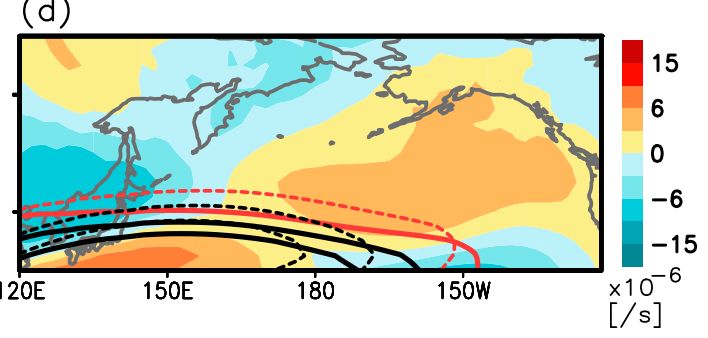

FIG. 12. Isotherms for mean temperature of westernmost NPO models (solid lines) of (a) IPSL-CM5B-LR (model 21) and (b) MIROC5 (model 22) and ensemble averaged values for the NPOwest models (dashed lines; 5-K interval) at $700-\mathrm{hPa}$ level common in (a) and (b). Red isotherms $(255 \mathrm{~K})$ are for easy comparison between the counterparts. Shading represents the difference in the mean temperature gradient $(|\nabla \bar{T}|)$ between each model and NPOwest group (NPOwesternmost minus NPOwest). (c),(d) As in (a) and (b), but for isotachs of mean zonal wind at $300 \mathrm{hPa}$ (Contours are drawn at 30,40 , and $50 \mathrm{~m} \mathrm{~s}^{-1}$ ). Shading denotes the difference in the mean wind stretching deformation $(\partial \bar{u} / \partial x-\partial \bar{v} / \partial y)$.

of the NPO, but further analysis on the temporal evolution of the NPO is required to confirm this relation.

Nevertheless, the above point is further supported by examining the mean state characteristics of the two models with the westernmost NPO, IPSL-CM5B-LR (model 21) and MIROC5 (model 22). As shown in Figs. $12 \mathrm{a}$ and $12 \mathrm{~b}$, the two models exhibit unique features in climatological trough, the atmospheric baroclinicity, and jet stream. Specifically, the continental troughs in these two models exhibit very steep slope (the solid lines) compared to the NPOwest group (the dashed lines). This distinct climatological planetary wave structure leads to a noticeably weak mean baroclinicity over the North Pacific (shading), which is also reflected in the nearly constant temperature gradient in Fig. 6e (the blue solid curves). Note that the temperature gradient of the other models peaks at around $160^{\circ} \mathrm{E}$ (the orange and gray curves in Fig. 6e). This prominent characteristic of the mean thermal field may be the cause of the weak NPO-like variability of these models revealed by the distinct EOF2 and EOF3 patterns.

We find that observation also provides evidence (Fig. 13). During a period between 1965 and 1984 (the black curve), the mean thermal field had a low and nearly constant gradient in the zonal direction, similar to the results of IPSLCM5B-LR (model 21) and MIROC5 (model 22). This period is part of $\mathrm{P} 2$, and thus the mean temperature gradient that is lower than the $\mathrm{P} 2$ average (the red dotted line) reflects interdecadal variation in the mean field within the $\mathrm{P} 2$. Interestingly, the EOF2 for this 20-yr period (Fig. 13b) exhibits double northern centers that are similar to the EOF2 of IPSL-CM5B-LR (model 21) and MIROC5 (model 22). The consistency between the observations and the model results suggest that the tripolar EOF2 patterns indicating weak NPO-like variability may be an atmospheric response incidental to the distinct low and flat distribution of the mean temperature gradient.

On the other hand, with regard to the mean jet structure in the two westernmost NPO models (Figs. 12c,d), southward shrinkage of the jet is found in the downstream. This horizontal distribution is contrasting to the northward expansion of the jet in NPOeast group models (Fig. 6d). However, we could not find any common features in the mean wind stretching deformation of these models (shading), which again supports the vital role of APE conversion in the development of the NPO. Likewise, the mean bias in MRI-CGCM3 (model 28) might affect the reproducibility of NPOlike variability as the leading EOF mode. As denoted by the blue dotted curve in Fig. 6e, the model has a mean temperature gradient that is far below average. This distinct background condition is presumed to be related to the atypical eigenvector pattern of this model. However, to verify this, investigation encompassing the 

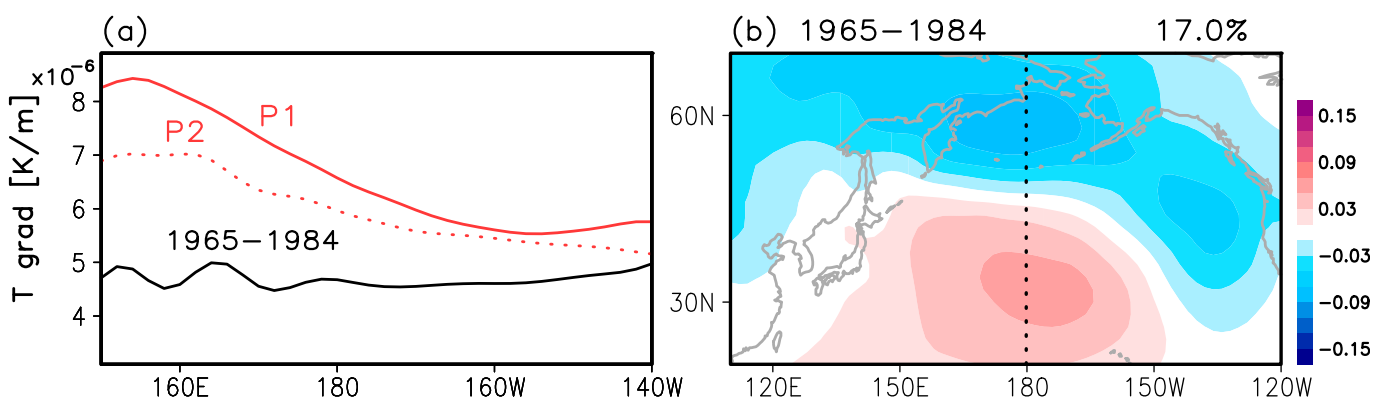

FIG. 13. (a) Mean temperature gradient over the $40^{\circ}-55^{\circ} \mathrm{N}$ meridional range for $\mathrm{P} 1$ (red solid curve), P2 (red dotted curve), and 1965-84 (the black curve). (b) Observational EOF2 patterns for the 1965-84 period. The black dotted line represents the date line.

three-dimensional structure of the climatological wave is required.

\section{Summary and discussion}

Based on recent advances in the understanding of the dynamics of the NPO, we investigated the spatial characteristics of the NPO simulated in the piControl experiments using CMIP5 models and their relationship with the climatological mean states in these climate models. It is known that the maintenance of the NPO largely depends on the conversion of APE from the mean thermal field through the heat exchange across the steep temperature gradient between the cold Eurasian continent and the warm North Pacific (Tanaka et al. 2016). Barotropic KE conversion from the climatological jet is also revealed to positively contribute to the maintenance of the NPO. The crucial role of APE and $\mathrm{KE}$ conversions suggests that changes in the mean thermal field and mean wind characteristic can lead to substantial changes in the NPO. It was found that changes in the baroclinicity of the mean flow are associated with the zonal position of the NPO's northern center by modulating APE conversion (Sung et al. 2019). Motivated by earlier results, we compared the mean temperature gradient and mean wind stretching deformation in the CMIP5 models with a particular focus on the region where APE and KE conversions strongly affect the position of the NPO. The majority of the models, which reasonably capture the typical features of the NPO, exhibited a clear linear relationship between the temperature gradient in the climatological mean state and the zonal position of the NPO, and likewise in the results of mean wind deformation. In other words, the simulated NPO exhibited a distinct eastward shift in its northern center if the model mean state had a climatological continental trough over the North Pacific that expanded more to the east. In addition, it was identified that some models whose EOF2 patterns did not reflect the typical features of the NPO had a large bias in the mean thermal field. This suggests that a large mean state bias in a climate model hinders the precise simulation of the climate mode and subsequent climate extremes because thermal and hydrological extremes in North America can be in part attributable to the NPO (Baxter and Nigam 2015; Lee et al. 2015; Lin et al. 2017; Linkin and Nigam 2008).

Although this study focused on the spatial structure of the NPO, the results suggest a possible modulation of the mean state in determining the intensity of the NPO, because the stronger mean baroclinicity in the NPOeast group models can supposedly intensify the NPO anomaly, not only affecting the zonal position. Indeed, average intensity of the NPO in the NPOeast group was identified to be stronger than in the NPOwest group (data not shown). Besides, the westernmost NPO group also shows relatively weaker intensity of the NPO. However, the observational result does not seem to follow this relation. As found in Chen et al. (2018), the observed NPO is overall weaker than the simulated intensity. But, as we showed in Figs. 6e and 6f, the magnitudes of the observational mean temperature gradient and mean wind deformation are almost comparable to those of CMIP5 models. It seems that the intensity of the NPO is determined by more complex processes, and further analysis is required for more precise understanding of this issue.

Another important question raised from this study is whether the climatological planetary wave condition also affects other leading modes in addition to the NPO. To verify this possibility, we repeated the same analysis for the first leading EOF patterns of the seasonal SLP anomalies in the CMIP5 models, which capture interannual variation in the Aleutian low intensity (not shown). The EOF1 pattern is supposed to represent the regional branch of the Pacific-North American (PNA) pattern (Wallace and Gutzler 1981). Our preliminary analysis suggests that, unlike the 
EOF2, the EOF1 does not show notable relationship with changes in the background state in the North Pacific (now shown).

The finding of this study has implications regarding future projections of the NPO. While piControl experiments are examined here to determine the inherent characteristics of the NPO, earlier work by Chen et al. (2018) evaluated future projected changes in the NPO under warming conditions. By comparing data between the historical and RCP8.5 runs, Chen et al. found a slight difference in the ensemble means of the NPO pattern. The ensemble average of the RCP8.5 experiments identified a slight enhancement of the northern pressure anomaly of the NPO to the east compared to historical experiments, possibly indicating that future warming may facilitate the shift of the NPO eastward. Although the difference was not great, it would be worth addressing it further in detail by assessing quantitative changes in the mean field in each climate model.

The past observational changes may also hint about the future change. The Aleutian low has intensified during the past twentieth century, which is perceived to result from the El Niño-like warming in the tropical Pacific (Gan et al. 2017). Recent study also reveals that colder (warmer) decadal condition in the tropical eastern Pacific provides background condition favorable for eastward (westward) shift of the NPO by modulating climatological planetary wave over the extratropical North Pacific (Sung et al. 2019). Therefore, the westward shift of the NPO during P2 may correspond in part to the background changes induced by the tropical mean state. Average features of the NPOeast group in CMIP5 models show relative cooling over the eastern tropical Pacific relative to the NPOwest models. However, the intermodel composite difference does not match very well with the observed pattern in Sung et al (2019). This is possibly because mean biases in CMIP5 models result from various factors apart from the tropical condition (not shown).

The present results highlight the usefulness of investigating the energetics of atmospheric modes to understand their intrinsic characteristics and changes resulting from changes in the background flow. Because the NPO straddles a region of strong baroclinicity, its energetics are distinct in that they favor processes related to APE and KE conversions. In this regard, the linear relationship identified in our study between the NPOs in the CMIP5 models and background baroclinicity may represent a natural causal relationship. Therefore, a similar approach may provide insight into the behaviors of other climate modes simulated using climate models. For example, changes in the continental trough over the
North Atlantic Ocean and the consequent change in climate modes would be worthy of investigation.

Acknowledgments. We thank three anonymous reviewers for their constructive comments. This research was supported by National Research Foundation of Korea (NRF) grants funded by the South Korean government (MSIT) (NRF-2018R1A5A1024958 and 2019 R1C1C1003161). Author Sung was supported by NRF2018R1D1A1B07044112. SWY was funded by the Korea Meteorological Administration Research and Development Program under Grant KMI2018-03211. Author Kosaka is supported by the Japan Society for Promoting Science (Grant 18H01278) and Japan Ministry of Education, Culture, Sports, Science and Technology through "Arctic Challenge for Sustainability" and "The Integrated Research Program for Advancing Climate Models."

\section{REFERENCES}

Baxter, S., and S. Nigam, 2015: Key role of the North Pacific Oscillation-west Pacific pattern in generating the extreme 2013/14 North American winter. J. Climate, 28, 8109-8117, https://doi.org/10.1175/JCLI-D-14-00726.1.

Chen, Z., B. Gan, L. Wu, and F. Jia, 2018: Pacific-North American teleconnection and North Pacific Oscillation: Historical simulation and future projection in CMIP5 models. Climate Dyn., 50, 4379-4403, https://doi.org/ 10.1007/s00382-017-3881-9.

Compo, G. P., and Coauthors, 2011: The Twentieth Century Reanalysis Project. Quart. J. Roy. Meteor. Soc., 137, 1-28, https://doi.org/10.1002/qj.776.

Gan, B., L. Wu, F. Jia, S. Li, W. Cai, H. Nakamura, M. A. Alexander, and A. J. Miller, 2017: On the response of the Aleutian low to greenhouse warming. J. Climate, 30, 39073925, https://doi.org/10.1175/JCLI-D-15-0789.1.

Holton, J. R., 2004: An Introduction to Dynamic Meteorology. Academic Press, 535 pp.

Kosaka, Y., and H. Nakamura, 2010: Mechanisms of meridional teleconnection observed between a summer monsoon system and a subtropical anticyclone. Part I: The Pacific-Japan pattern. J. Climate, 23, 5085-5108, https://doi.org/10.1175/2010JCLI3413.1.

Lee, M.-Y., C.-C. Hong, and H.-H. Hsu, 2015: Compounding effects of warm sea surface temperature and reduced sea ice on the extreme circulation over the extratropical North Pacific and North America during the 2013-2014 boreal winter. Geophys. Res. Lett., 42, 1612-1618, https://doi.org/10.1002/2014GL062956.

Lin, Y.-H., L. E. Hipps, S.-Y. S. Wang, and J.-H. Yoon, 2017: Empirical and modeling analyses of the circulation influences on California precipitation deficits. Atmos. Sci. Lett., 18, 19-28, https://doi.org/10.1002/asl.719.

Linkin, M. E., and S. Nigam, 2008: The North Pacific Oscillationwest Pacific teleconnection pattern: Mature-phase structure and winter impacts. J. Climate, 21, 1979-1997, https://doi.org/ 10.1175/2007JCLI2048.1.

Nakamura, H., M. Tanaka, and J. M. Wallace, 1987: Horizontal structure and energetics of Northern Hemisphere wintertime teleconnection patterns. J. Atmos. Sci., 44, 3377-3391, https:// doi.org/10.1175/1520-0469(1987)044<3377:HSAEON>2.0.CO;2. 
Pak, G., Y.-H. Park, F. Vivier, Y.-O. Kwon, and K.-I. Chang, 2014: Regime-dependent nonstationary relationship between the East Asian winter monsoon and North Pacific Oscillation. J. Climate, 27, 8185-8204, https://doi.org/10.1175/JCLI-D-1300500.1 .

Rogers, J., 1981: The North Pacific Oscillation. Int. J. Climatol., 1, 39-57, https://doi.org/10.1002/joc.3370010106.

Sung, M.-K., H.-Y. Jang, B.-M. Kim, S.-W. Yeh, Y.-S. Choi, and C. Yoo, 2019: Tropical influence on the North Pacific Oscillation drives winter extremes in North America. Nat. Climate Change, 9, 413-418, https://doi.org/10.1038/s41558 019-0461-5.

Tanaka, S., K. Nishii, and H. Nakamura, 2016: Vertical structure and energetics of the western Pacific teleconnection pattern J. Climate, 29, 6597-6616, https://doi.org/10.1175/JCLI-D-150549.1.
Wallace, J. M., and D. S. Gutzler, 1981: Teleconnections in the geopotential height field during the Northern Hemisphere winter. Mon. Wea. Rev., 109, 784-812, https://doi.org/10.1175/ 1520-0493(1981)109<0784:TITGHF $>2.0$. CO;2.

Wang, L., W. Chen, and R. Huang, 2007: Changes in the variability of North Pacific Oscillation around 1975/1976 and its relationship with East Asian winter climate. J. Geophys. Res., 112, D11110, https://doi.org/10.1029/2006JD008054.

Wang, X., M. Chen, C. Wang, S.-W. Yeh, and W. Tan, 2019: Evaluation of performance of CMIP5 models in simulating the North Pacific Oscillation and El Niño Modoki. Climate Dyn., 52, 1383-1394, https://doi.org/10.1007/s00382-018-4196-1.

Yeh, S.-W., D.-W. Yi, M.-K. Sung, and Y. H. Kim, 2018: An eastward shift of the North Pacific Oscillation after the mid1990s and its relationship with ENSO. Geophys. Res. Lett., 45, 6654-6660, https://doi.org/10.1029/2018GL078671. 